\title{
Gender Dysphoria: A Review Investigating the Relationship Between Genetic Influences and Brain Development
}

This article was published in the following Dove Press journal: Adolescent Health, Medicine and Therapeutics

\author{
Ferdinand JO Boucher (D) \\ Tudor I Chinnah (D) \\ University of Exeter, Medical School, St \\ Luke's Campus, Exeter, EXI 2LU, UK
}

Correspondence: Ferdinand JO Boucher Email fb339@exeter.ac.uk

\begin{abstract}
Gender dysphoria (GD) is a facet of modern human biology which is believed to be derived from the sexual differentiation of the brain. GD "involves a conflict between a person's physical or assigned gender and the gender with which he/she/they identify", as defined in the DSM-5. Individuals report feeling uncomfortable and faced with prejudice from those around them, affecting their mental health. Elucidating the relationship between genetic influences on gonadal and brain development could give an insight into understanding this clinical condition. To explore this issue, a review of the literature database was carried out. Evidence suggests that abnormal biological processes, including mutations in certain genes, can lead to abnormal gonadal development, causing some fetuses to present with indifferent gonads and to be reassigned at birth to the default female sex. This disparity in genetic influences relates to an increased likelihood of a diagnosis of GD. An investigation into complete androgen insensitivity syndrome, involving androgen receptor (AR) gene mutation, suggests that such individuals also experience GD. It is known that the brains of males and females are different. Evidence further suggests that brain anatomy and neuronal signaling pathways are more closely aligned with a person's perceived gender identity. Individuals who present with discordant gonadal and brain developments experience psychological challenges that may contribute to a state of unease or generalized dissatisfaction with their biological sex. These point to a possible biological and genetic underpinning of GD as stemming from a discordance between gonadal and brain development. However, not enough evidence has associated these differences with GD. Further research is required to elucidate the true mechanisms and possible inheritance pattern of GD for a better education and greater understanding by clinicians and the general public on perceptions regarding GD. Keywords: sex, gender, gender dysphoria, gonadal development, brain development
\end{abstract}

\section{Plain Language Summary}

Gender identity is an important issue in society, yet its causative mechanism is poorly understood. The main anatomical differences between males and females are the genitalia and the brain. This paper reviewed the literature to explore the link between genetic influences and brain development, and their impact on gender identity. Investigating these developmental mechanisms could lead to advances in the understanding of gender dysphoria, a condition whereby an individual's gender and biological sex are mismatched. Exploring the biochemical development of the genitalia highlights the differences between males and females, notably how testosterone elicits the pathways of male development in an embryo. When research has been conducted in people with Androgen Insensitivity Syndrome, a condition where the testosterone receptor is mutated and faulty, and thus cannot function, 
gender dysphoria is observed as the body is genetically male but anatomically female. It is known that the structure of male and female brains differs; it is found that people with gender dysphoria have a brain structure more comparable to the gender to which they identify. The review of the literature suggests that there is a disparity between the brains of those who identify differently to their assigned gender at birth, highlighting a multifactorial underpinning of the gender identity. Further research is required to shed light on the molecular mechanism of this, allowing for greater education and understanding of this scientific and social phenomenon.

\section{Introduction}

Gender dysphoria is a nosological entity defined when used as a diagnosis under the Diagnostic and Statistical Manual of Mental Disorders (DSM-5) and the American Psychiatric Association (APA). It is a general descriptive term referring to an individual's discontent with the assigned gender. ${ }^{1}$ In this context, it is important to highlight the difference between sex and gender - two terms which are commonly used interchangeably and yet mean very different things. The biological sex of an individual refers to a person being either male or female based on their chromosomal makeup and their genitalia. ${ }^{2}$ It is used in a social, medical and legal context to "categorize" people under the two sexes, as men or women. This term is assigned at birth and often stays with a person for the rest of their life. The term gender is harder to define, as it is to do with how someone identifies, how a person fits in with the social norms, activities and attributes that are commonly associated with men and women. ${ }^{3}$ Whilst for many people sex and gender go in unison, for some, there is an imbalance between anatomical body structure and self-perception. A collective term for these individuals is "transgender", whilst others will relate to terms such as gender dysphoria (GD) or gender incongruence. This is not to do with the individuals' sexual orientation, but rather to do with their sexual identity. Sexual orientation "refers to an enduring pattern of emotional, romantic and/or sexual attraction to men, women or both sexes". 4 Sexual identity is a component of one's personal identity, encompassing moral, ethical, religious beliefs in the development of a multi-dimensional identity. ${ }^{5}$

A stricter definition requires that GD individuals suffer anxiety from their condition. However, it is important to point out that true GD individuals suffer emotionally and psychologically, with suicide rates in this population being significantly above average. It has been suggested that up to $41 \%$ of transgender people attempt suicide compared to $5 \%$ in the general population. ${ }^{6}$ This provides a moral impetus for conducting further research into the causes of the condition in order to gain a fuller understanding and develop more advanced and appropriate support and/ or treatment aiming to improve the quality of life for those with GD.

Transgender is an umbrella term that stands in place for a range of individuals whose gender identity diverges from that which they were assigned at birth. Whilst the global awareness of this term has grown, certain communities have elaborated a wider plethora of terms for individuals who identify along non-binary lines. ${ }^{7}$

To address their anxiety, these individuals decide to either change their gender socially or seek reconstructive surgery with hormone-therapy to tailor their body image to their desired sex. Although specific details as to the mechanisms behind GD have been elucidated, a complete causal understanding has not been elaborated. Nonetheless, it is accepted that an array of social factors plays a role in mapping a GD individual's self-perception on the gender scale. These include, but are not exclusive to, the environment in which children are brought up, the pronouns used to refer to the individual, the media's perspective and the manner in which they speak about the topic.

The differences between the (two) sexes have been a surviving concept for over 200 years, and yet it is still in doubt as to the correlation of these differences to the structure and function of the human brain. ${ }^{8}$ Men and women have been shown to differ in many characteristics, most noticeably with regards to gender identity: men perceive themselves as male whilst women perceive themselves as female.

This paper explores the underlying biology which determines the sex that a person is assigned at birth and the correlation between the effects of sex genes and hormones on the development of the brain and the gonads. It focused on genetic conditions where these do not develop in unison, compared the "normal" and "abnormal" development in GD. Finally, conclusions were drawn on where the limits of biological understanding of GD currently lie and present several areas for possible research to further elucidate the condition.

\section{Materials and Methods}

Sources used were drawn from PubMed, NHS Digital databases and relevant publicly available online resources, 
including World Health Organization. Search terms used include "gonadal development", "fetal brain development", "gender", "SRY" and "testosterone". To refine the search, specific terms such as "diagnosis" and "abnormalities" were also used. These terms were used to make a Boolean algorithm. Non-English articles were excluded to ensure consistency of relevant terminology. All referenced articles were read in full. A specific focus was put on articles within the last 20 years as this field of science and psychology is novel, although older papers were reviewed when concerning a first discovery. Only research articles that were focused on human research were selected, with materials on non-human animals being disregarded.

\section{Discussion}

\section{The Role of Sex-Determining Genes in the Sexual Differentiation of Human Gonads}

Sexual differentiation is a multi-factorial process that involves many genes, both autosomes (non-sex chromosomes) and allosomes (sex chromosomes). Sexual differentiation can be defined as the event or process leading to the development of testes or ovaries, starting from "indifferent" or "bipotential" genital precursors. 9 The initial (indifferent) stage of gonadal development arises at week 7 of embryonic development. Although the sex of the embryo is determined at fertilization, the gonads do not morphologically change until later. The initial stages of differentiation are common, but the effects of transcription factors later play a crucial role in changing the path of gonadal differentiation. At the start of differentiation, males and females have two pairs of ducts, but through the action of transcription factors further gonadal differentiation commences to proliferate or be suppressed depending on the genetically determined sex at the time of fertilization. Male development depends primarily on the mesonephric ducts (also known as the Wolffian Ducts), which develop during development of the urinary system, whereas the paramesonephric ducts (also known as the Müllerian ducts) are lost in the male but become central in female development.

It is the SRY protein, a transcription factor which is the expression product of the sex-determining region of the Y chromosome (SRY) gene located on the Y chromosome, which provides the driving force for sexual differentiation of the mammalian gonads. The subsequent differentiation of the indifferent gonads resolves around the presence, or absence, of this gene. When this protein is not produced, no masculinization of the embryo ensues, and the body develops into the female phenotype. It is not until the ninth week of development where external genitalia can be distinguished, such that a mutation in this gene can be visualized. It, therefore, follows that the female sex is the "default" sex, as both male and female gonads develop from a common female primordial gonad, as summarized in Figure 1. This is the reason that testing for the sex of

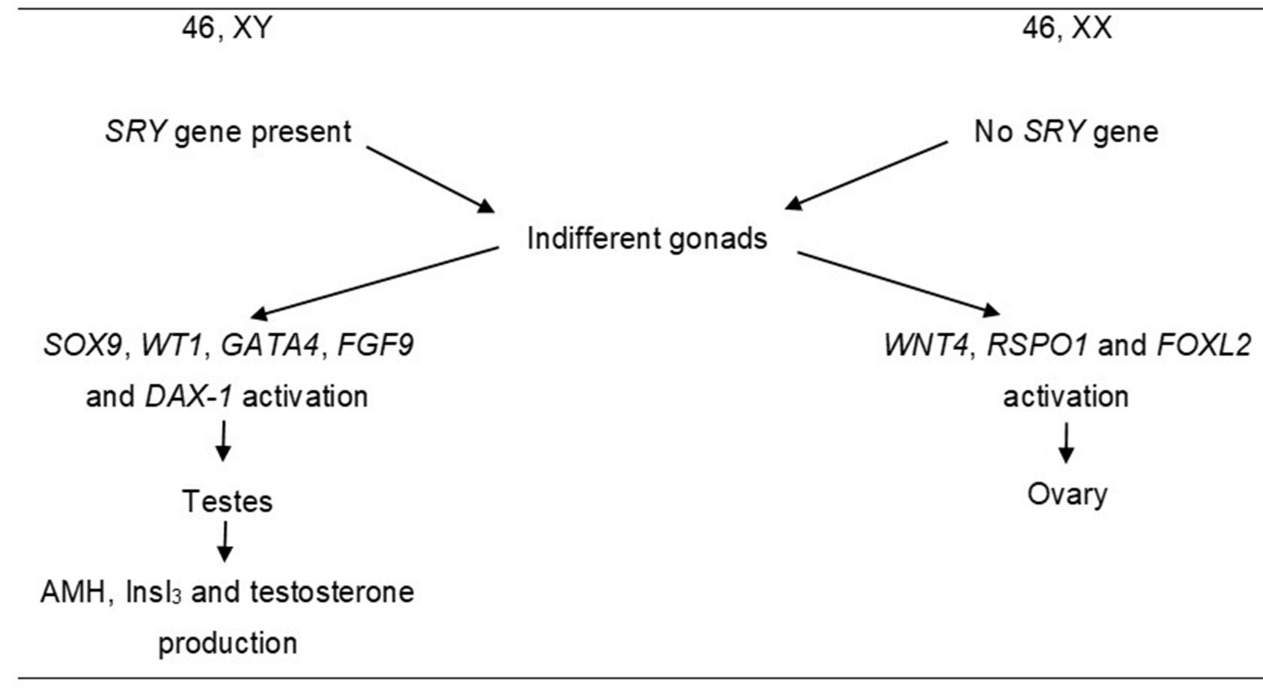

Figure I Influence of the SRY gene on indifferent gonads. Influence of the SRY gene on indifferent gonads. A summary figure highlighting the importance of the SRY gene, whereby its presence dictates the outcome of differentiation of the gonads. In individuals who have this gene, normally located on the $Y$ chromosome, testes development will occur. In the gene's absence, ovaries will form. 
a fetus (if desired or required) is not done until the 20week stage.

\section{Testes Development}

If the primordial cells are genetically male, they will be carrying an $\mathrm{X}$ and $\mathrm{Y}$ chromosome. Following activation of the $S R Y$ gene, the primordial testes found in the urogenital ridges develop two key cell types: Leydig cells and Sertoli cells. The developing testes also secrete androgens, the male hormones, most importantly, testosterone. It is through the action of testosterone, secreted by the Leydig cells, binding to androgen receptors (AR), that differentiation of the mesonephric ducts is initiated and this in parallel stabilizes the developmental cascade. ${ }^{10}$ Structures such as the efferent ductules and the epididymis of the testes, the vas deferens and the ejaculatory duct originate from these ducts. Following maturation and adult development, it is these structures that store and produce sperm.

Precursor gonadal cells first express steroidogenic factor 1 (Sf1). ${ }^{11}$ Its release causes the activation of the $S R Y$ gene, which initiates the biological process of sexual differentiation. Sf1 interacts with the SRY protein (through reciprocal activation) and the SRY-Box transcription factor 9 (SOX-9) transcription factors. The SRY gene product causes activation of the SOX-9 gene. This increase in expression causes pre-Sertoli cells to differentiate and expand. The SRY protein then supports gonad differentiation into Sertoli cells following an increase in Sfl activity. The Sertoli cells secrete and regulate the synthesis of antiMüllerian hormone (AMH, also known as Müllerian inhibiting substance). ${ }^{12}$ Following secretion of $\mathrm{AMH}$ from these cells, the paramesonephric ducts degenerate and further inhibit the development of female characteristics, most noticeably the fetal reproductive tracts. ${ }^{13}$ As noted above, Leydig cells are then recruited and start producing testosterone around the 9th week of gestation. ${ }^{13}$

The importance of testosterone should be noted as the key hormone in this process of differentiation, allowing the cascade of reactions to occur and normal male genitals to arise. If there is a mutation in one or more of the genes responsible for this cascade, causing for instance the presence of a faulty receptor or reduced secretion of testosterone, errors in development will occur.

\section{Ovarian Development}

If the primordial cells are genetically female, they will be carrying two $\mathrm{X}$ chromosomes and no $\mathrm{Y}$ chromosomes (therefore importantly no $S R Y$ gene). With the absence of testosterone and $\mathrm{AMH}$, the paramesonephric ducts differentiate into the structures from which the uterine tubes and the upper portion of the vagina develop. These ducts descend and fuse with each other to form the uterovaginal primordium which, when in contact with the dorsal wall, develop into the paramesonephric tubercle. This singlelayered epithelium layer gives rise to various layers within the vagina, including the ciliated columnar epithelium in the uterine tube and the stratified squamous epithelium. ${ }^{14}$ The mesonephric ducts degenerate due to a lack of testosterone production.

Much less is known about the process of differentiation of ovarian than testicular development, but several genes have been identified as having a regulatory effect in this fetal stage of development. In the "pro-ovary" cells, with the absence of the Y chromosome, there is a decrease in Sf1 activity due to an absence of SRY protein. The activation of Wingless-related integration site (Wnt4) gene and R-spondin1 (Rspol) gene, two other transcription factors that form part of the Wnt signaling pathway, suppresses the activity of Sox $9 .^{15}$ This process is then followed by expression of Forkhead box L2 (Foxl2) gene, further hindering the activity of the $\operatorname{Sox} 9$ transcription factor. The latter then inhibits the differentiation of Sertoli and Leydig cells. Following this cascade, somatic support cells differentiate into follicle cells which, in combination with oocytes, develop into primordial ovarian follicles.

It has been shown that mutations in the Rspol gene, which no longer inhibit the expression of Sox9, could lead to the development of XX testicular disorders of sexual development (DSD). ${ }^{16}$ This proves the importance of the Rspol gene in the sexual determination of an individual, highlighting that the $S R Y$ gene is not solely responsible for this process, as summarized in Figure 2. It would be of great scientific interest to conduct a follow-up study on this piece of research, identifying whether this mutation or a similar abnormal genetic makeup influences the participants identified gender.

\section{The Role of Sex-Determining Genes in the Sexual Differentiation of the Human Brain}

There are multiple biological differences between males and females, from anatomy to susceptibility to various diseases. It is known that the anatomical structure of the female and male brain is different, not only in a structural perspective but also hormonal. It is also proven that the 


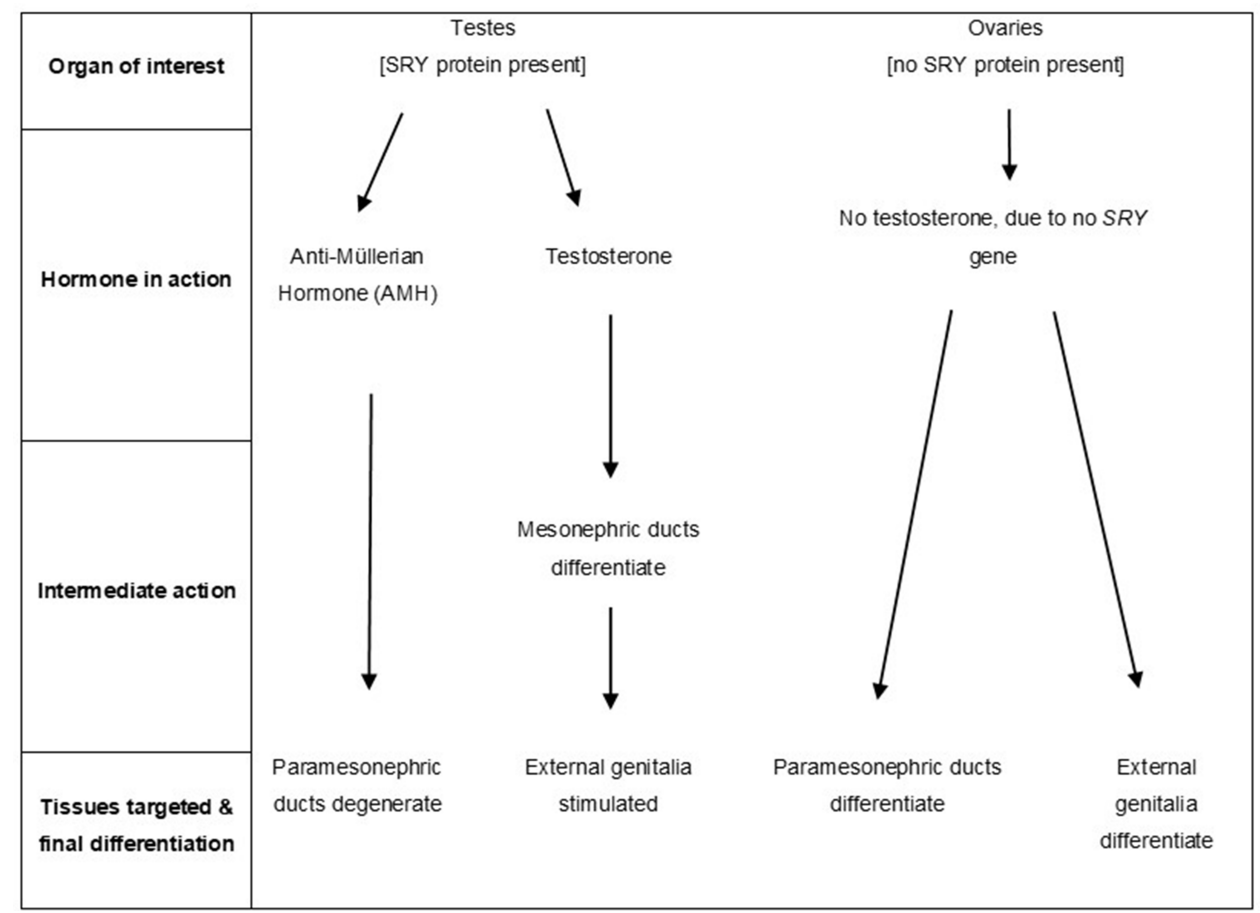

Figure 2 Sexual differentiation of the gonads following activation of SRY gene on tissues. A summary of the hormones produced, and which tissue develop following their effect on the sex organs.

size of brains in the two sexes is different, although this is predominantly due to the average larger body size of males. ${ }^{17}$ It is hypothesized that individuals who identify as a different gender to their anatomical sex have a brain structure resembling that of the sex they are perceived to be.

This is based on the idea that gender is decided in the womb. The brain forms the central part of the central nervous system (CNS) - a system that develops in the second trimester of fetal development. The steroid hormones playing a role in gonadal development have long been studied and shown to elicit an effect on the brain's sexual development. ${ }^{18}$ These fetal hormones initiate a cascade of effects leading to a change in the size of various regions of the brain, the number of nerve cells and the relative quantity of neurotransmitters. ${ }^{18}$

It is important to distinguish between cause and effect, which is especially challenging when looking at the anatomy of the brain. It is of great scientific and medical interest to explore whether it is the anatomical structures of the brain that may lead to a person identifying with gender dysphoria or whether it is the thought patterns that influence brain anatomy. An insight into whether gender identity is a biological and chromosomal-based condition, or is it brought about by environmental factors such as influences through society and culture or an epigenetic mechanism will lead to a better understanding of how we can support GD individuals in our community. A further complexity in elucidating cause and effect in studies on individuals with GD is that use of hormone therapy, a common route for treatment could further alter the brain structure.

\section{Anatomical and Neurostimulation Differences in Cisgender Adults}

Cisgender individuals are those whose gender identity matches the sex that they were assigned at birth. Male brain sexual differentiation is believed to originate during gestation and the perinatal period, during which two peaks of gonadal hormones are observed. From the time of puberty onwards, sex hormones will affect and map the previously established neuronal patterns.

It has been shown that males have a higher mean volume in their hippocampus (involved in spatial memory and in consolidating information from short-term to longterm memory), their amygdala (playing a role in processing emotions) and the thalamus (involved in relaying motor and sensory signals), although the data presented appear to have a degree of overlap. This was noted when the authors investigated the overall and subcortical brain 
volumes, which allowed them to look at the differences between the two sexes. Whilst the male results were further distributed to the right, indicating a higher volume, $48.1 \%$ of the data points appeared in the overlap region. ${ }^{19}$ Furthermore, men have been shown to have a higher concentration of androgen than of the estrogen receptor, whilst women have a larger hippocampus with greater levels of estrogen hormone and lower concentrations of the AR. ${ }^{20}$ A recent study showed that men's brains were on average $8-13 \%$ larger than their female counterparts. This confirmed previous findings that suggest that males have larger total brain volume, cerebrum and cerebellum volumes $\left(10 \%\right.$ and $9 \%$, respectively). ${ }^{21}$ In contrast, females have higher density left frontal lobe and larger volumes in the right frontal lobe.

These results confirm an asymmetric development of the brain. There are therefore clear anatomical differences between the brain structure of males and females. This could be visualized on a scale, with the two sexes forming the extremities of it. People experiencing GD appear to not be restricted to a single category or extremity, but rather occupy a middle ground. Although the biological development of this pattern at the fetal stage has yet to be explored, it raises questions as to the importance of sex-determining genes and hormones and their impact on brain development. It could be hypothesized that there is a hormonal imbalance in those who experience GD, an imbalance that is not perfectly confined to just one sex. In addition, environmental factors are likely to elicit an effect, as are nutrition, exposure to sunlight, and stress, all of which may alter brain function. $^{22}$

\section{The Brain Under Gender Dysphoria Hypothalamic Response}

Androstadienone is an odorous artificial steroid that structurally resembles androgens. Savic et al suggested that smelling the drug induced a hypothalamic response in heterosexual females but not in heterosexual males. ${ }^{23}$ They measured the cerebral activity through regional cerebral blood flow with a positron emission tomography. Whilst the authors state they investigated twenty-four healthy subjects, twelve females and twelve males, they do not divulge whether these were cisgender individuals or not. The range in the results produced could be further investigated if the gender of the individuals had been investigated, potentially finding some variability in the genders that the individuals identify with. Further investigation comparing this methodology and the results in transgender individuals will be of great scientific interest to further clarify the neurological response that individuals have in line with their perceived gender.

A more recent study backed these findings, utilizing a range of androstadienone concentrations and measuring the hypothalamic response in men's and females' brains. ${ }^{24}$ It was seen that females experienced a significantly higher response to the drug than men, further supporting previous results. Although the data may not yet be conclusive, noting that a biological mechanism that explains these data is yet to be elucidated. However, this technique provides a good method whereby researchers can distinguish between individuals who identify as either males or females. These findings also have wider ramifications as they provide some evidence that there is a biochemical explanation for attraction between the two sexes.

This simple and "objective" experimental measure has been extrapolated to be used in studies involving transgender people in order to elicit a hypothalamic response which may indicate their identified gender. ${ }^{25}$ A recent study conducted on gender dysphoric teenagers found that individuals with GD showed a hypothalamic response more like that of their perceived gender rather than their natal sex. The controls, the non-GD individuals, responded in line with their birth sex; however, the pre-adolescent GD birth girls responded in an unclear way whilst the preadolescent GD birth boys responded like boys. ${ }^{26}$ These results further highlight a difference in neurostimulation between the two sexes. These results have been further confirmed in individuals who had undergone genderaffirming surgery, finding that the cognitive, emotional and sensorimotor regions of their brains align with that of their perceived gender. ${ }^{27}$

\section{Structure of the GD Brain}

Findings from neuroimaging studies provide evidence suggesting that the structure of the brains of trans-women and trans-men differs in a variety of ways from cis-men and cis-women, respectively, as indicated below. Looking at the volume of the cortex using voxel-based morphometry, Simon et al found that females and male-to-female gender dysphoric individuals had a smaller grey matter volume in their left somatosensory and primary motor cortices in comparison to cisgender male controls. ${ }^{28}$ These findings reflect a female-like cortical expression in those who identify as females, unrelated to their anatomical sex. It is however noted that the small sample size $(n=17)$ 
provides only weak evidence to support the finding and as such the results should be taken with caution.

A recent study investigated the volume of grey matter in individuals with GD and found that they had a smaller volume in the left posterior superior hemisphere of the cerebellum compared to male controls and a smaller volume of the right inferior orbitofrontal cortex compared to female controls. ${ }^{29}$ Although these areas are proven to express high levels of sex hormones and steroid receptors, this study does not permit the determination of the source of these findings, whether they are due to endocrine, environmental, genetic influences or a combination of these factors.

\section{Heredity and Gender Dysphoria}

It is important to establish the extent to which gender dysphoria might have a genetic component or even be a genetic condition, ie, one that can be attributed to genetic factors alone, or whether it is all or partly to do with environmental factors (including hormone levels during puberty and psychological factors). Heritability studies provide insight into which conditions or biological traits exhibit a heritable aspect. Heritability is measured on a scale of 0 to 1 , where 0 defines no genetic explanation and 1 defines complete genetic determination. Most of the studies investigating gender dysphoria have been conducted on twins, and less so from family studies.

In a study of GD involving 1891 twins, an inheritance pattern of $0.50-0.57$ in men and $0.30-0.37$ in women was reported. ${ }^{30}$ This study was focused on childhood gender dysphoria but involved twins with the median age of 29 which leaves open the possibility of recall bias. There have since been a handful of studies investigating this trait further. One of these studies found that genetics accounted for $62 \%$ of the variance in gender dysphoria; ${ }^{31} \mathrm{Knafo}$ et al found an inheritance pattern of 0.21 in boys and 0.74 in girls ${ }^{32}$ and Burri et al found a female inheritance pattern of $0.11,{ }^{33}$ indicating a very low influence of genes. However, the authors mention a high chance of error which is plausible as the rate is significantly lower than in previous studies. Although studies have been conducted investigating the importance of genetics in GD, none of these investigated specific genes.

At present no single gene has been identified to have a strong correlation to the development of GD. The Cytochrome P450 17A1 (CYP17) gene, which controls the level of sex hormones being produced, has a mutated allele (A1) which is more frequent in transgender men. ${ }^{34}$
Although minimal research has been conducted in this field to this date, this could be a prominent field of further study in the hope of finding a genetic mechanism underpinning or associated with GD.

The importance of the SRY, AR and CYP17 genes in their respective pathways in embryological development cannot be disputed. It is with interest that these commonly accepted biological pathways are being explored in an ever-growing psychological and sociological phenomenon, specifically GD.

\section{Androgen Insensitivity Syndrome}

DSD is a generalized term regrouping a range of syndromes and disorders which affect the sexual development of an individual. Common examples of these include congenital adrenal hyperplasia, androgen insensitivity syndrome (AIS) and 5-alpha-reductase deficiency. This review investigates AIS into greater detail due to the lack of formally published and accepted diagnostic criteria. With a range of diagnostic measurers presented in individuals with AIS, on a molecular, morphological and biochemical basis, AIS provides an insight into the difficulty in diagnosing appropriately.

AIS is a rare genetic condition whereby androgens do not elicit the intended effect and is a subset of DSD. ${ }^{35}$ AIS is due to a mutation or deletion in the $A R$ gene, causing an inability of androgens to elicit their effect on tissues preand post-birth. ${ }^{36}$ These individuals will be genetically male but express a female phenotype. Their genitalia at birth can be ambiguous, and if so tend to be reassigned to the female sex for ease of surgery. The ambiguous genitalia are more commonly observed in those with partial or mild form of the condition. Mutations in these genes are also apparent in trans women, the cytosine-adenineguanine (CAG) repeats. ${ }^{37}$ It is found that trans women have this repeat sequence significantly longer than their cisgender male counterparts. However, further research ought to be carried out investigating the extent of other variants in the sex hormone signaling genes, and their correlation with gender dysphoria.

This highlights the importance of androgens throughout the process of sexual differentiation, and that a single mutation in a gene encoding a protein operating upstream of the process will cause irreversible changes to the phenotype of the individual. It is seen that individuals with AIS have a varying amount of androgen expressivity, leading to two subtypes of AIS: either complete (CAIS) or partial (PAIS) androgen insensitivity syndrome. 


\section{Complete Androgen Insensitivity Syndrome}

With androgens completely blocked from being able to play a role, all tissues, including the brain, are unable to respond to testosterone. XY individuals will have a female phenotype and have a female gender identity. This is because the mesonephric ducts regress and male genitalia do not develop. The paramesonephric ducts do not develop due to the presence of AMH. A clinical presentation of an individual with CAIS would be phenotypically female, seen from birth, amidst normal serum male androgen levels. At adolescence, there is normal breast development and pubertal growth, but primary amenorrhea is noted.

A case report of a 41-year-old female revealed absence of internal female organs and presence of testes, alongside a "normal" female body figure. ${ }^{35}$ The androgens produced in these females are converted to estrogens through the action of aromatase enzymes. This further promotes development of secondary female characteristics, including bone maturation. ${ }^{36,38}$ A recent study involving 113 female patients with CAIS found a decreased level of bone mineral density in comparison to control females. This study further investigated this initial finding by comparing age of gonadectomy, removal of the internal testes, but found no statistical difference. ${ }^{39}$ This is a clinically valuable piece of research as it frees CAIS women from being governed by a deadline by which they must undergo surgery. This study was carried out in a single practice, and although the researchers ensured heterogeneity in their sample of participants, the study design does raise the risk of potential bias via the list of participants who chose to enroll. Another found limitation with this study is that all participants had CAIS, none having any other form of DSD, such as PAIS. It would be of interest to investigate whether this lack of significant change in bone mineral density is observed across a spectrum of conditions.

\section{Partial Androgen Insensitivity Syndrome}

A diagnosis of PAIS will largely depend on the extent to which phenotypic expression of external female genitalia, in response to AR function, is seen. There appears to be a spectrum of mutations leading to a diagnosis of PAIS, with a clinical presentation depending on the individual's genitalia response to androgens. ${ }^{36}$

Ambiguous genitalia are often seen in a clinical setting, with patients presenting with mild to severe hypospadias, a condition whereby the opening of the urethra is on the inferior aspect of the penis. Patients have also been recorded to present with a micropenis. Although extensive metabolic research must be carried out before a confirmed diagnosis is made, sex reassignment surgery is done at birth to create female genitalia as a first-line treatment.

Although these individuals are genetically male, with an XY makeup, they are brought up as girls following female-typical social roles. Although from a biological perspective it is clearly seen that the female sex is the common/default path of fetal development, this is often not the case post-birth where societal pressures and expectations can play very significant roles in the development of gender identity. For example, it is commonly held that society imposes a degree of "guidance" or pressure on an individual's developing gender identity merely based on his or her physical appearance. It is not known what the presumably reduced psychological impact would be on these individuals if society were somehow prevented from imposing stereotypically fixed gender roles and thoughts on children based on physical characteristics.

Putting aside such questions as might be raised by a consideration of the social scientific context, and from a purely natural scientific perspective (based on the study of AIS individuals and the known importance of AR in the development of normal male brains), it is reasonable to hypothesize that an increased androgen expressivity in the brain plays a role in the development of gender identity. ${ }^{40}$

There is an ongoing debate amongst clinicians as to the use of surgery to remove the testes in patients suffering from CAIS or PAIS, particularly to do with the time and the technical aspects of the surgery. This debate incorporates many physical, legal and psychological aspects that are not further investigated here. However, from a medical point of view, a proven increased risk of malignancy of the testes has been shown if they are retained within the patient. ${ }^{41,42}$ Also, their removal reduces the risk of genderidentity problems arising in teenage years or adulthood as no confounding genitalia is present. ${ }^{41}$ Although there is a consensus which has been established that the testes should be left intact until the child's teenage years, presumably to allow informed consent from the individual, it is, however, reasonable to expect that some families will commit to the surgery early in the life of the child. This might be for religious reasons, family history of disease, or simply personal preference. Nonetheless, a multidisciplinary decision must be taken to ensure the best outcome for the patient, involving not only clinicians but also psychologists and social workers to support the family in making the best decision for their child. This irreversible surgery poses risks to the psychological state of the 
individual, as androgens and various products of the Y chromosome are still present and active. Surgery tends to include removal of the testes but can also include vaginal surgery if the dilation is minimal or unsuccessful.

The prospect of knowing that one is "part male and part female" can pose ongoing GD issues for the individual having undergone surgery, even if physically the individual is only identifiable by one sex alone. The ageold consensus of evidence-based medicine does not apply here. In these individuals, with evidence suggesting both sexes, the chromosomal makeup and phenotype act as contradicting variables: the decision of gender identity is not clear cut. This raises major hurdles leading to a range of unanswered questions, regarding the application and time of surgery or regarding the postoperation support offered to GD individuals, which most likely do not have a set answer. Late diagnosis (or a deferral of surgery) is preferred as the individual can then make an informed and autonomous decision about their own health based on how they self-identify. The age of consent in the United Kingdom is set at 16 from the General Medical Council. ${ }^{43}$ The minimum age is set at this time point as it is believed that it ensures attainment of appropriate levels of cognitive function and allows the person to consider all pillars of ethics in their decision-making.

\section{Further Research}

These discussions highlight an unknown but complex etiology of GD. It must be acknowledged that GD is a multi-factorial condition and that genetics alone does not fully account for the phenomenon. Although there appears to be a degree of genetic underpinning, in common with other medical conditions, non-genetic factors such as social upbringing, wealth, access to education and diet may also contribute to this condition. Psychology could also play a role in this sexual differentiation, a parent-child interaction or anxiety predisposition could impact on the way a GD child perceives themselves and affect the normal development of the brain.

There is a lack of knowledge of the influences of sexual identity on neuronal function. ${ }^{4-46}$ There has been little research on people identifying as transgender and how their brain structure differs from those whose sex and gender are matched. It is of great importance to further research into this field [ie, how their brain structure/function differs from those whose sex and gender are matched], going beyond the observed anatomical structures of the brain to understand how the brains of people with GD correlate with those whose sex and gender are united, whilst also providing insight into the role that society has on the behavior of people with GD.

Many psychiatric conditions differ in frequency between men and women. This being the case, it is important to understand the biochemistry of the brain that explains why the risk is increased in either sex. For example, the number of females diagnosed with Alzheimer's disease is greater than the number of males. ${ }^{47}$ This finding is supported by a recent publication by E.E. Sundermann, which delves further into the biomechanical aspect of the disease and the involvement of the $\varepsilon 4$ allele which is known to play a key role in Alzheimer's onset. ${ }^{48}$ Although clear findings are reported, with females being higher at risk, there is a lack of explanation as to the differences that account for this at a genetic level which provides a mechanism underpinning this sexual difference. This could be a field for further research, notably involving individuals who have changed sex, to investigate whether their risk of Alzheimer's disease is linked to their biological sex at birth or their gender identity. Research shows that brain structure correlates more strongly to gender identity than it does to biological sex. If the disease risk is correlated to the structure of the brain, and thus gender, this could promote personalized medicine and help healthcare professionals tailor their care towards patients based on their gender and not their biological sex.

With further research into this field, some light could be shed as to the origins of GD and how it affects the brain function. With a better understanding of the condition and improved education of clinicians and the general public alike, support and awareness could be offered to individuals who identify as an opposing gender to their genetic sex and the suffering associated with GD may thereby be reduced.

\section{Conclusion}

The studies and research that have been conducted allow us to confirm that masculinization or feminization of the gonads does not always proceed in alignment with that of the brain development and function. There is a distinction between the sex (visible in the body's anatomical features or defined genetically) and the gender of an individual (the way that people perceive themselves).

It is noted that the causal mechanism of GD is unknown, but the importance of biological influences via genes and hormones is clear. Individuals with AIS present with a spectrum of gonadal appearance, which cannot be 
categorized as either completely male or female but maintain their typical function. This duality, in addition to individuals who present with discordant gonadal and brain developments, creates psychological challenges that may contribute to a state of unease or generalized dissatisfaction with their biologically assigned gender. A focus on brain anatomy highlights a difference between males and females and shows that brain function too is gender-orientated, with, for example, a hypothalamic response to androstadienone which differs significantly between the two sexes. In this test, individuals with GD have a hypothalamic response more like that of their experienced gender, rather than their genetic sex. These point to a possible biological and genetic underpinning of GD as stemming from a dissonance between gonadal development and brain sexual differentiation and orientation. However, GD is a multifactorial condition which biology alone cannot fully explain. Further research is required to identify a true causal mechanism of GD, as well as investigating an inheritance pattern. With further research and the resulting increase in awareness of the condition, including better education of clinicians and understanding in the lay population, there may be a greater acceptance of individuals who identify as a different gender to their anatomical sex and thereby contribute to a step forward in modern society.

\section{Author contributions}

All authors made a significant contribution in the conception, study design, execution, data acquisition, analysis and interpretation and took part in drafting, revising, critically reviewing and revising the article; gave final approval of the version to be published; have agreed on the journal to which the article has been submitted; and agree to be accountable for all aspects of the work.

\section{Disclosure}

We can confirm that this manuscript has not been published previously either in whole or in part and is not under consideration for publication by any other journal. No participation of human subjects was involved in the study and ethics permission was not required. All authors have no potential competing financial interests regarding the submitted article and have read and accepted responsibility for the manuscript's contents.

\section{References}

1. American Psychiatric Association. Diagnostic and Statistical Manual of Mental Disorders. 5th ed. 2013.
2. World Health Organization. Gender and human rights. Published 2018. Available from: https://www.who.int/reproductivehealth/ topics/gender_rights/sexual_health/en/. Accessed July 9, 2020.

3. World Health Organization. Gender. Published 2018. Available from: http://www.who.int/gender-equity-rights/understanding/genderdefinition/en. Accessed July 9, 2020.

4. American Psychiatric Association. Sexual orientation and homosexuality. Published 2008. Available from: https://www.apa. org/topics/lgbt/orientation. Accessed July 9, 2020.

5. Luyckx K, Schwartz SJ, Goossens L, Beyers W, Missotten L. Processes of personal identity formation and evaluation. In: Handbook of Identity Theory and Research. 2011:77-98. doi:10.1007/978-1-4419-7988-9_4

6. Williams A. Risk factors for suicide in the transgender community. Eur Psychiatry. 2017;41(S1):s894. doi:10.1016/j.eurpsy.2017.01.1820

7. American Psychological Association. Defining transgender terms. September 2018.

8. Goodman N. The descent of man and selection in relation to sex. J Anat Physiol. 1871;5(Pt2):363-372.

9. Utiger RD. Sexual differentiation. Encyclopædia Britannica, inc. Published 2018. Available from: https://www.britannica.com/ science/sexual-differentiation. Accessed July 9, 2020.

10. Hannema SE, Print CG, Charnock-Jones DS, Coleman N, Hughes IA. Changes in gene expression during Wolffian duct development. Horm Res. 2006;65(4):200-209. doi:10.1159/000092408

11. Luo X, Ikeda Y, Parker KL. A cell-specific nuclear receptor is essential for adrenal and gonadal development and sexual differentiation. Cell. 1994;77(4):481-490. doi:10.1016/00928674(94)90211-9

12. Sekido R, Lovell-Badge R. Sex determination involves synergistic action of SRY and SF1 on a specific Sox9 enhancer. Nature. 2008;453(7197):930-934. doi:10.1038/nature06944

13. Morel Y, Roucher F, Mallet D, Plotton I. Genetic of gonadal determination. Ann Endocrinol. 2014;75(2):32-39. doi:10.1016/j. ando.2014.04.005

14. Schoenwolf GC, Bleyl SB, Brauer PR, Francis-West PH. Larsen's Human Embryology. 2015. doi:10.1016/B978-0-443-068119.10011-9

15. Ohnesorg T, Vilain E, Sinclair AH. The genetics of disorders of sex development in humans. Sex Dev. 2014;8(5):262-272. doi:10.1159/ 000357956

16. Parma P, Radi O, Vidal V, et al. R-spondin1 is essential in sex determination, skin differentiation and malignancy. Nat Genet. 2006;38(11):1304-1309. doi:10.1038/ng1907

17. Luders E, Toga AW. Sex differences in brain anatomy. In: Progress in Brain Research. 2010:2-12. doi:10.1016/B978-0-444-536303.00001-4

18. De Bellis MD. Sex differences in brain maturation during childhood and adolescence. Cereb Cortex. 2001;11(6):552-557. doi:10.1093/ cercor/11.6.552

19. Ritchie SJ, Cox SR, Shen X, et al. Sex differences in the adult human brain: evidence from 5216 UK biobank participants. Cereb Cortex. 2018;28(8):2959-2975. doi:10.1093/cercor/bhy109

20. Halpern DF. Sex Differences in Cognitive Abilities. 4th ed. Psychology press; 2013. doi:10.4324/9780203816530

21. Ruigrok ANV, Salimi-Khorshidi G, Lai MC, et al. A meta-analysis of sex differences in human brain structure. Neurosci Biobehav Rev. 2014;39:34-50. doi:10.1016/j.neubiorev.2013.12.004

22. Wachs TD, Georgieff M, Cusick S, Mcewen BS. Issues in the timing of integrated early interventions: contributions from nutrition, neuroscience, and psychological research. Ann N Y Acad Sci. 2014;1308 (1):89-106. doi:10.1111/nyas.12314

23. Savic I, Berglund H, Gulyas B, Roland P. Smelling of odorous sex hormone-like compounds causes sex-differentiated hypothalamic activations in humans. Neuron. 2001;31(4):661-668. doi:10.1016/ S0896-6273(01)00390-7 
24. Burke SM, Veltman DJ, Gerber J, Hummel T, Bakker J. Heterosexual men and women both show a hypothalamic response to the chemo-signal androstadienone. PLoS One. 2012;7(7):e40993. doi:10.1371/journal.pone.0040993

25. Berglund H, Lindström P, Dhejne-Helmy C, Savic I. Male-to-female transsexuals show sex-atypical hypothalamus activation when smelling odorous steroids. Cereb Cortex. 2008;18(8):1900-1908. doi:10.1093/cercor/bhm216

26. Burke SM, Cohen-Kettenis PT, Veltman DJ, Klink DT, Bakker J. Hypothalamic response to the chemo-signal androstadienone in gender dysphoric children and adolescents. Front Endocrinol. 2014;5:60. doi: $10.3389 /$ fendo. 2014.00060

27. Schneider MA, Spritzer PM, Minuzzi L, et al. Effects of estradiol therapy on resting-state functional connectivity of transgender women after gender-affirming related gonadectomy. Front Neurosci. 2019;13:817. doi:10.3389/fnins.2019.00817

28. Simon L, Kozák LR, Simon V, et al. Regional grey matter structure differences between transsexuals and healthy controls - A voxel based morphometry study. PLoS One. 2013;8(12):e83947. doi:10.1371/ journal.pone.0083947

29. Hoekzema E, Schagen SEE, Kreukels BPC, et al. Regional volumes and spatial volumetric distribution of gray matter in the gender dysphoric brain. Psychoneuroendocrinology. 2015;55:59-71. doi:10.1016/j. psyneuen.2015.01.016

30. Bailey JM, Dunne MP, Martin NG. Genetic and environmental influences on sexual orientation and its correlates in an Australian Twin sample. J Pers Soc Psychol. 2000;78(3):524-536. doi:10.1037/00223514.78.3.524

31. Coolidge FL, Thede LL, Young SE. The heritability of gender identity disorder in a child and adolescent twin sample. Behav Genet 2002;32(4):251-257. doi:10.1023/A:1019724712983

32. Knafo A, Iervolino AC, Plomin R. Masculine girls and feminine boys: genetic and environmental contributions to atypical gender development in early childhood. J Pers Soc Psychol. 2005;88 (2):400-412. doi:10.1037/0022-3514.88.2.400

33. Burri A, Cherkas L, Spector T, Rahman Q. Genetic and environmental influences on female sexual orientation, childhood gender typicality and adult gender identity. PLoS One. 2011;6(7):e21982. doi:10.1371/journal.pone.0021982

34. Fernández R, Cortés-Cortés J, Esteva I, et al. The CYP17 MspA1 polymorphism and the gender dysphoria. J Sex Med. 2015;12 (6):1329-1333. doi:10.1111/jsm.12895

35. Ozdemir O, Sari ME, Akmut E, Selimova V, Unal T, Atalay CR. Complete androgen insensitivity syndrome with a large gonadal serous papillary cystadenofibroma. J Hum Reprod Sci. 2014;7 (2):148-150. doi:10.4103/0974-1208.138875
36. Hughes IA, Davies JD, Bunch TI, Pasterski V, Mastroyannopoulou K, Macdougall J. Androgen insensitivity syndrome. Lancet. 2012;380(9851):1419-1428. doi:10.1016/S01406736(12)60071-3

37. D'Andrea S, Pallotti F, Senofonte G, et al. Polymorphic cytosine-adenine-guanine repeat length of androgen receptor gene and gender incongruence in trans women: a systematic review and meta-analysis of case-control studies. J Sex Med. 2020;17 (3):543-550. doi:10.1016/j.jsxm.2019.12.010

38. Almeida M, Laurent MR, Dubois V, et al. Estrogens and androgens in skeletal physiology and pathophysiology. Physiol Rev. 2017;97 (1):135-187. doi:10.1152/physrev.00033.2015

39. King TFJ, Wat WZM, Creighton SM, Conway GS. Bone mineral density in complete androgen insensitivity syndrome and the timing of gonadectomy. Clin Endocrinol. 2017;87(2):136-140. doi:10.1111/cen.13368

40. Fernández R, Guillamon A, Cortés-Cortés J, et al. Molecular basis of gender dysphoria: androgen and estrogen receptor interaction. Psychoneuroendocrinology. 2018;98:161-167. doi:10.1016/j.psyne uen.2018.07.032

41. Mouriquand PDE, Gorduza DB, Gay CL, et al. Surgery in disorders of sex development (DSD) with a gender issue: if (why), when, and how? J Pediatr Urol. 2016;12:139-149. doi:10.1016/j.jpurol.2016.04.001

42. Lee PA, Nordenström A, Houk CP, et al. Global disorders of sex development update since 2006: perceptions, approach and care. Horm Res Paediatr. 2016;85(3):158-180. doi:10.1159/000442975

43. General Medical Council. Making decisions. Published 2018. Available from: https:/www.gmc-uk.org/ethical-guidance/ethical-guidance-fordoctors/0-18-years/making-decisions. Accessed July 9, 2020.

44. Beery AK, Zucker I. Sex bias in neuroscience and biomedical research. Neurosci Biobehav Rev. 2011;35(3):565-572. doi:10.1016/ j.neubiorev.2010.07.002

45. Kaczkurkin AN, Raznahan A, Satterthwaite TD. Sex differences in the developing brain: insights from multimodal neuroimaging. Neuropsychopharmacology. 2019;44(1):71-85. doi:10.1038/s41386018-0111-z

46. Rigby N, Kulathinal RJ. Genetic architecture of sexual dimorphism in humans. J Cell Physiol. 2015;230(10):2304-2310. doi:10.1002/ jcp. 24979

47. Mazure CM, Swendsen J. Sex differences in Alzheimer's disease and other dementias. Lancet Neurol. 2016;15(5):451-452. doi:10.1016/ S1474-4422(16)00067-3

48. Sundermann EE, Tran M, Maki PM, Bondi MW. Sex differences in the association between apolipoprotein E $\varepsilon 4$ allele and Alzheimer's disease markers. Alzheimer's Dement Diagnosis, Assess Dis Monit. 2018. 10:438-447. doi:10.1016/j.dadm.2018.06.004
Adolescent Health, Medicine and Therapeutics

\section{Publish your work in this journal}

Adolescent Health, Medicine and Therapeutics is an international, peer-reviewed, open access journal focusing on health, pathology, and treatment issues specific to the adolescent age group. All aspects of health maintenance, preventative measures and disease treatmen interventions are addressed within the journal and practitioners from all disciplines are invited to submit their work as well as healthcare researchers and patient support groups. The manuscript management system is completely online and includes a very quick and fair peerreview system. Visit http://www.dovepress.com/testimonials.php to read real quotes from published authors. 